\title{
Network Externality Products C \& Competition Strategy: An Experimental Economics Approach
}

\author{
Geng Zhou, Jiaofei Zhong, Han Zhou and Yu Chen \\ Information school, Renmin University of China \\ 100872 Beijing, China \\ hxk19l@163.com
}

\begin{abstract}
Based on the theory of Complexity Adaptive System (CAS) and methods of experimental economics, using Multi-Agent-Based Simulation, we build a model of the network externality product market with the platform Netlogo3.1.2, analyze the macroscopic consumers' behavior, find out the important aspects that influence the spreading of products and the proper strategies of enterprises during this process and make a prediction of the China's instant messenger software market.
\end{abstract}

\section{Introduction}

Network externality is an essential rule of network. It has been a vital aspect in the construction of network since network was born.

In the IT industry, by taking advantage of network externality, a large number of enterprises (like Microsoft, Tencent and many web2.0 websites) succeeded through high-speed growth and hold their position with enormous profit unthinkable for enterprises in other fields, while by failing to make use of it, an even larger number of enterprises lost their chance forever. Since the spreading of network externality products is dynamically complex, using the theory of Complexity Adaptive System (CAS), multi-agent modeling and experimental economics methods to analyze the procedure has a special significance. Through the singleproduct-market model, we find that the 3 aspects of network externality, information circulation rate and initial number of users have an obvious impact on the spreading of network externality products and we also explore the proper occasion to release advertisements. And through the two-products-model, we explore the effect of random factors, and the strategies to fight against adverse situation. Finally, we make a prediction of the fractionized China's instant massager market. 


\section{Research background}

\subsection{Network externality}

Network externality is also called the "network effect". If the value of a product to a user is decided by the total number of users, we say this product possesses the property of "network externality". This kind of "externality" has a tight relation with the scale of network: the few members in a network not only have to bear the high cost of operating, but also can commute information with only a few people; as the network grows the cost for every user decreases and the scope of communication is broadened, so that every user gains a higher value.

The Metcalfe law describes the effect: the value of a telecommunications network is proportional to the square of the number of users of the system $\left(n^{2}\right)$. [1]

\subsection{Agent-based modeling}

ABM (Agent-based modeling) is widely adopted in the research of complex adaptive systems comprising of large number of active agents such as society, economics, and environment. Network externality product market is a typical complex adaptive system. A network externality product comes into a market, spreads among the customers, competes with its competitors until winning or washed out. This is a typical complex procedure in a complex system. $\mathrm{ABM}$ is eligible in analyzing such problems.

\section{Objective of research}

Use multi-agent modeling, theory of Complexity Adaptive System(CAS), and experimental economics methods to simulate and analyze the procedure of spreading of the network externality products, so as to demonstrate and explore the economical law, study the macroscopic behavior of consumers and the strategy of enterprises, find out the important aspects that influence the spreading and proper strategy to adopt to promote the spreading; use the conclusions gained from these to analyze and predict the China's instant massager software market.

\section{Descriptions of the model}

\subsection{Platform}

Operation system: Windows XP, Stimulation software: Netlogo 3.1.2, Statistics software: Excel 2003. 


\subsection{Structure of the model and agent behavior rule}

We made two models: a single product market model and a two products market model.

In both models we uses a two dimension environment to simulate the real market, forming a virtual commercial environment with more than 1000 agents (simulate the consumers) and a number of advertisements.

The rule in the model fall into 3 parts: motion rule, influence rule, buying rule. (1)Motion rule: every agent choose a random direction to move, the length of one step is a random number between 0 and the maximize number set according to the real market.

(2)Influence rule: Assume that the value of the network is in direct proportion with number of user agents (Metcalfe law); the influence from advertisements to agents and between agents increases as the network and network externality grows (certainly a more valuable network has a stronger impact).In both model, influence from buyers and advertisements to potential buyers will raise their "buying value"; in the two products market model, influence from advertisements to buyers will cause them to evaluate the opportunity cost and the switch cost when altering product.

(3)Buying rule: assume that there are already initial users when the model runs and all other agents are potential buyers (or potential users of free product) who can afford the cost. When a potential buyer contact with buyers and advertisements, it is influenced (as in the influence rule) and when the "buying value" reaches a certain value set according to the real market, the potential buyer will buy the product and turns into a buyer.

In the simulation of two products market, assume that there are two kinds of products competing with each other; they are similar, but not compatible with each other; and potential buyers choose only one of them. After a potential buyer buys a product, he is influenced by the advertisements, and will change product if he weighs opportunity cost and the switch cost and finds that he can gain more value by using the other kind of product.

\subsection{Figure of the concept model}

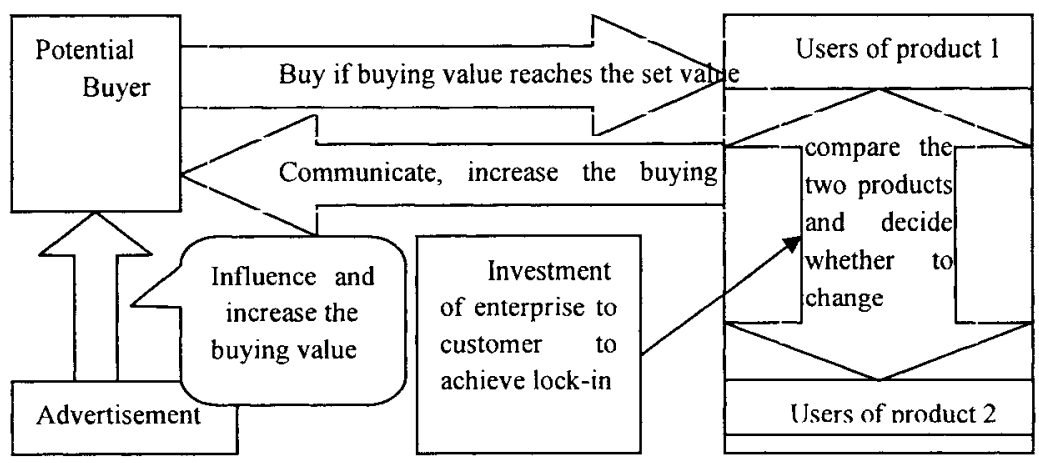

Figure1: 


\section{Analysis <1>: Single product market.}

The time and number in the model has no fixed units, they can be day/ten days/month/year and hundred/thousand/million according to the real market, and in the qualitative analysis here we care more about the proportion.

We will simulate a single product market (no competitors) here, aiming at studying the macroscopic behavior of the consumer and reaching the basic conclusions about the network externality product market. The results are as follows:

\subsection{Description of the curve of the growth in the number of users.}

Set the total number of agents as 1000 , initial users as 10 , value of network externality as 10 (highest value in this model), see figure 2 :

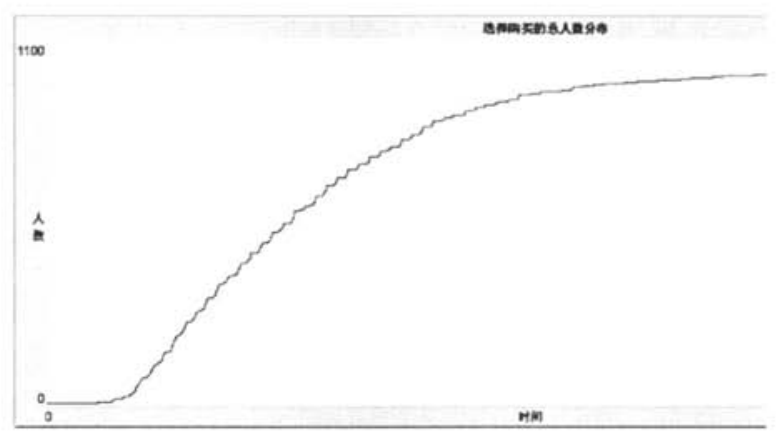

Figure2

From the curve, the number of users (buyers) goes through a period of accumulating, then it reaches the critical number, so that the value of the network is fully embodied and the influences from buyers and advertisements to potential buyers increase obviously, which then results in a time of rapid growth. When the market is nearly fully occupied, the growth rate decreases. At last the market is fully occupied.

\section{Case:}

Number of Tencent QQ instant massager contemporarily online users is as the figure3 [2] (original figure and curve draw according to it with Excel 2003): 


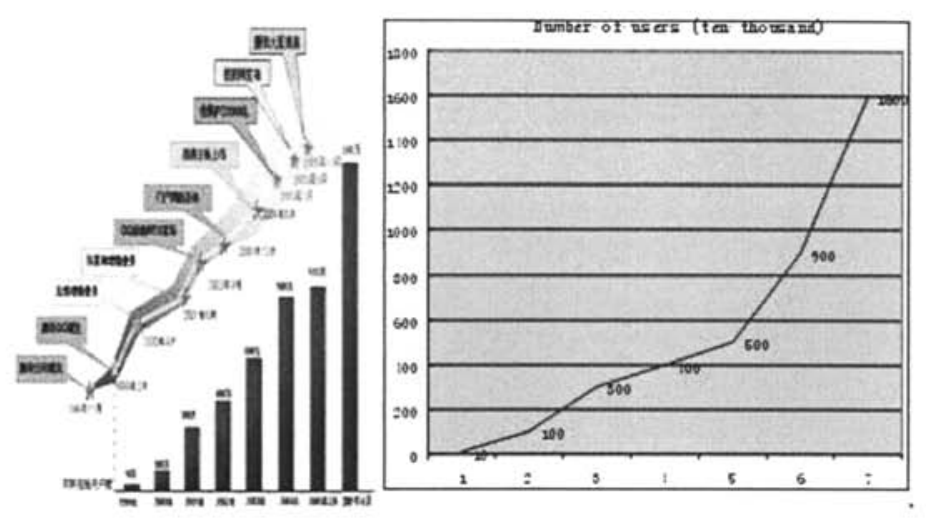

\section{Figure3}

It's easy to find that the number of QQ instant massager users is in the period of rapid growth, the critical number of high-speed growth is about 5 million.

Since there can't be an unlimited growth in the market of instant massager, it is can be predicted that after a short time of rapid growth, the increase rate will fall and the market will meet its bottleneck for the first time.

\subsection{The effects of network externality}

Set the total number of agents as 1000 , the initial users as 10 . The result is

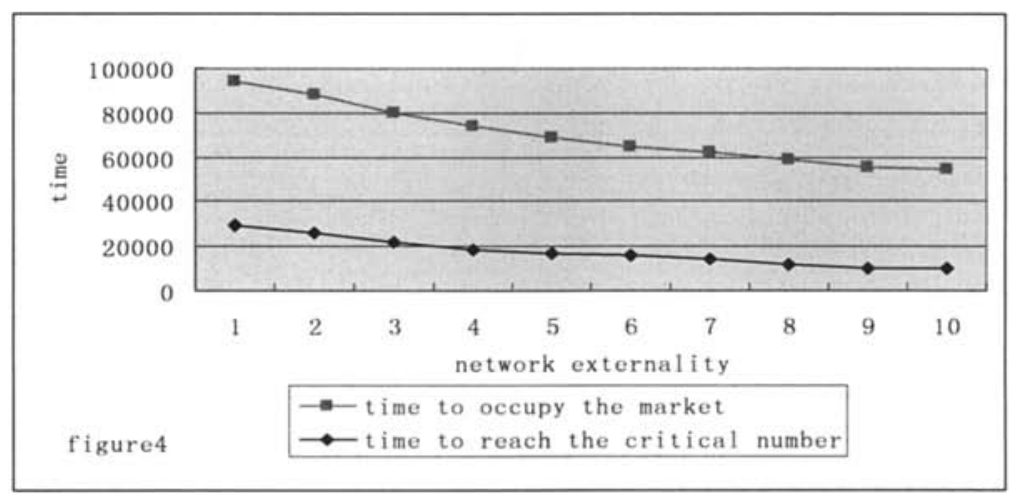

\section{Figure4}

By changing the value of the network externality (from 1-10) only, we see an obvious decrease in the time to reach the critical number and the time to occupy the market. There exists a faster growth in the high network externality markets than the low ones. The network externality of the product will form an efficient positive feedback between the growth in number and the growth in value of the network. And the market with higher network externality will make it earlier than the lower ones. 
Often the result of strong positive feedback is that only one winner enjoys most of the profits of the market.

\section{Competition strategy:}

1. For the high network externality products, the most important part is how to reach the critical number as early as possible so as to form the positive feedback and achieve an exponential growth in number of users ahead of the competitors. It'll help if there are a large number of initial users, and you can have them by excellent marketing, purchasing enterprise producing the same kind of products and so on. Also adopting effective marketing during the whole procedure will shorten the time to reach the critical number. Above all, attack first.

2. By increasing the network externality of the products will also shorten the time to reach the critical number and time to occupy the market. In the design of a product, enough attention should be paid to the functions which will raise the network externality.

\section{Case:}

According to the "2006 brief report on China IM" [3], the first three IMs used most are QQ $(80.1 \%)$, MSN (7.7\%), Tencent TM (1.6\%). Study shows that the IM industry ranks the third in the five industries which have the highest convergence. [4] We can see that Tencent QQ has monopolized the IM market. The Internet-based QQ was developed by Tencent Company in 1999.2, and by its high network externality register users of QQ grows rapidly. Tencent Company also combines it into several other correspondence platforms such as cell phone, which increase the network externality. Today QQ already has more than 580.5 million ID registered.[5] Besides, another reason for Tencent's success is that Tencent is a forerunner. It concentrates on IM very early and so gained the advantage of attacking first, thus achieved rapid growth the earliest.

\subsection{The importance of information circulation rate}

Set the total number of agents as 1000 , initial users as 10 , network externality as 10. See figure5:

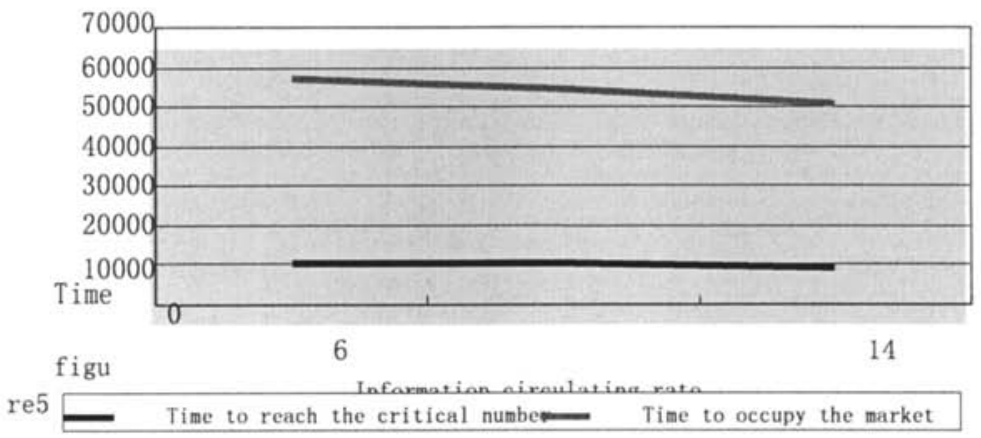

\section{Figure5}


When setting the information circulating rate as $2(4 \%$ of the highest value in the model), there is no such a period that the number of users grow rapidly. On the contrary, the figure is linear. See figure 6:

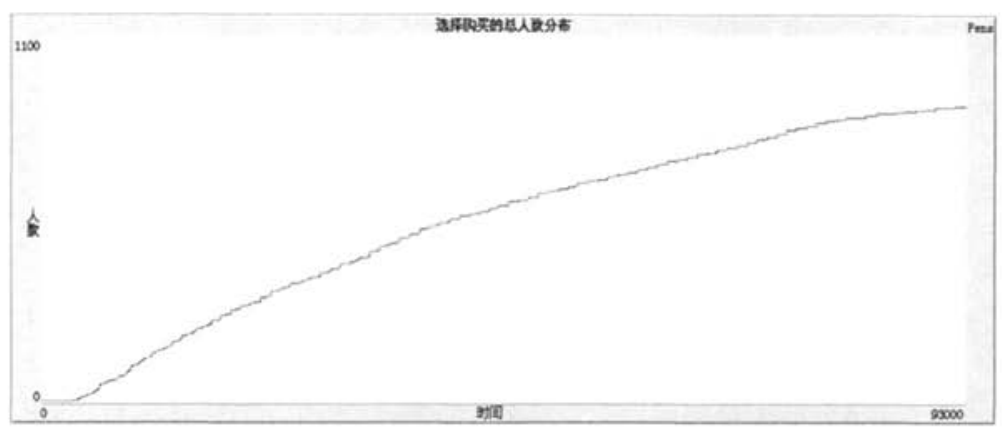

When the information circulating rate is extremely low, the information of buyers can not spread, thus they can't influence the potential buyers, therefore the growth of the value of the network can't be sensed by the potential buyers, and so the effect of network externality is quite low.

As the technique of correspondence improves, the rate of information circulation rises. By changing the value of information circulation rate (from 5 50) only, we see a decrease in the time to reach the critical number and to occupy the market. In fact, a higher information circulating rate decreases the time for the customers to evaluate and accept the product, and turn them into buyers quickly.

\section{Competition strategy}

1. It's better for enterprises to choose mediums which provides high information circulation rate such as the Internet and seriously consider introducing the product first in areas where the fundamental establishments related to information circulation rate are well-developed.

2. It will also help if the enterprises increase the information circulation rate themselves like building a product website or a search engine.

\subsection{The effect of initial number of users}

Set the total number of agents as 1000 , network externality as 10 and the information circulation rate as 50 . The result is figure7: 


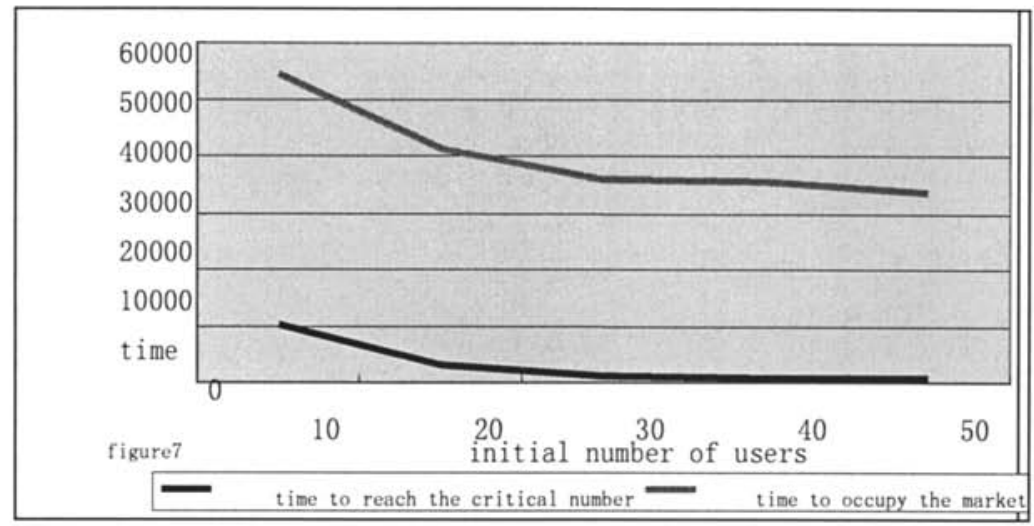

\section{Figure7}

By changing the value of initial users (from 5 50), we see an obvious decrease in the time to reach the critical number and to occupy the market. This is because the number of initial users is related directly to the time to reach the critical number.

\section{Competition strategy:}

Finance permitting, the enterprises should try hard to increase the initial number of users. Proper marketing strategy such as free edition, discount, binding with other products and excellent public relation will help. Purchasing enterprises producing the same kind of products will also help.

\subsection{Appropriate occasion to advertise}

We find that to stop advertising after the users have reached the critical number has little influence on the exponentially growth and the time to occupy the market. This is because after the critical number is reached, the influence from orally spreading and the behavior of buying increase greatly, and this kind of influence outweighs the influence of advertisements.

We know that few advertisements are put out to propagandize the basic function after the users of cell phone, telegrapher, BP reached the critical number.

\section{Competition strategy:}

After the high network externality product goes into the period of rapid growth, it is proper to lessen the advertisements on the basic function, and put the precious fund more on the value-added service so as to achieve a better lock-in (a higher swatch cost) on the buyers to increase the profit.

\section{Analysis $<2>$ : Two product market.}

There will always be competitors. The purpose of using this model to simulate a market with two network externality products(or enterprises) competing with each other is to go even nearer to the real market, and study the strategy of enterprises and behavior of consumers on a higher stage. Assume that the two products are 
similar(no essential difference) but not compatible with each other; and that the finance ability of the two enterprises are the same; the two products goes into the market at the same time.

\subsection{The influence of random factor}

Let both enterprises put out the same amount of advertisements and take no other action of competition.

Set the total number of agents as 1200 , network externality as 10 , and initial number of users as 10 (both 5). The result is figure8: (both of two curves represent the number of the buyers)

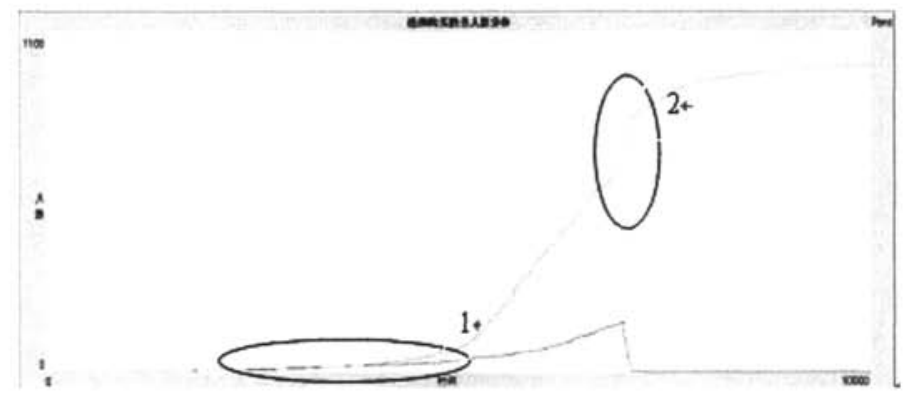

\section{Figure8:}

In ellipse 1 , in the beginning there occurs a small distinction in the user number, and then this small distinction made one product reach the critical number earlier and consequently resulted in a rapid growth. In ellipse 2 , the high value resulted from a large network of the product was fully realized. This value easily outweighs the value of the other product's network and the switch cost of the users of the other product so that the winning product attracted many users of the competitor's product. Those who changed their products along with the new buyer gave the number of the winning product "a big leap". At the same time, the losing of users caused a collapse of the competitor's product: the decrease of users and the decrease of value of the network formed a strong negative feedback which deprived the failing product of its users in no time.

Through experimenting many times, we found that competing in the same environment with no competition strategy besides advertisements, both enterprises could win, and the possibilities are almost the same. This shows the power of the random factor: there are some random factors in the market that influence the numbers of the early buyers of the products, and the distinction caused by this will provide one product a chance to reach the critical number earlier, thus resulting in an rapid growth and occupation of market.

\section{Competition strategy:}

1. Random factor of the market will cause a distinction in number of early buyers. If left unnoticed, the distinction will cause one product to reach the critical number of users earlier and make it the winner. 
2. It's advisable to be highly sensitive with the number of users and take actions to reach the critical number as early as possible whether you are lagging behind.

3 . The high network externality product market is usually a market with the winner seized the majority and achieves monopoly, while others-if not fail completelyshare the rest small part. Therefore, if an enterprise finds its adversary reaches critical number first and the gap between them can't be filled up quickly, it's time to consider seriously retreating from the market.

\section{2 fighting back}

Let the enterprise which lags behind take actions so that it may exceed its adversary.

Both put out their advertisements to the greatest extent.

Set the total number of agents as 1200 , network externality as 10 , and initial number of users as 10 (both 5). The result is figure9:

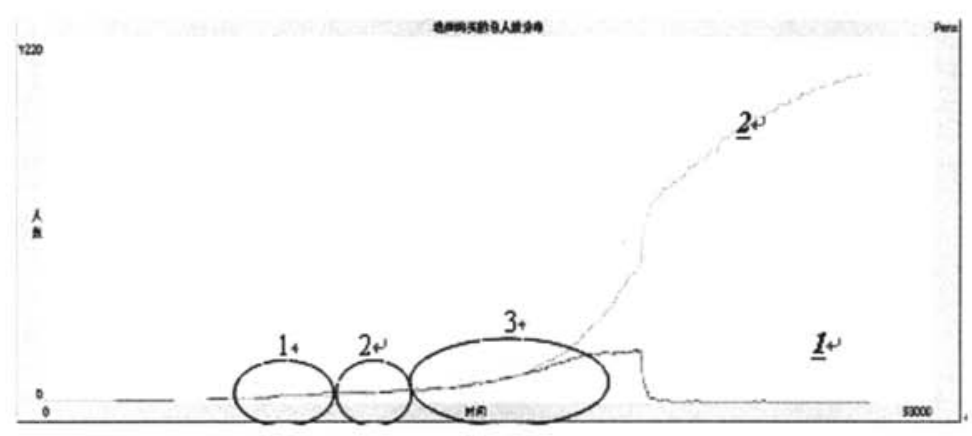

Figure9:

After the two products were introduced into the market, there exists a distinction in numbers of users in ellipse 1 caused by random factors, then enterprise $\underline{2}$ sensed the situation and raised its investment (includes two parts: the investment on buyers of enterprise 2 's product aiming at achieve lock-in; the investment on buyers of enterprise $\underline{\underline{l}}$ 's product aiming at helping them overcome the switch cost and make them change product.). We see shakes in ellipse 3 which shows that the new investment worked and caused some decrease in the users of product 1 by drawing them to the other side. Also the investment slowed the increase of new buyers of product 1 down by attracting the potential buyers who could be the buyers of product 1 if no action was taken. The result is enterprise $\underline{2}$ reached the critical number earlier and occupied the market. The adoption of action in time filled up the adverse gap and saved enterprise $\underline{\mathbf{2}}$.

\section{Competition strategy:}

1. During the spreading of high network externality products, once lagging behind, enterprise should take action to eliminate the gap immediately and achieve solid lock-in as early as possible. In the beginning, it's wise for enterprise to put more resource into increasing the number of users, and prepare enough funds for the following fierce battle to attract consumers. 
2. The importance of critical number will certainly make it the main battlefield. It's possible to take actions early and fight the adversary back successfully. Also it's a must to hold the favorable situation and enlarge the gap.

3. Although it's possible to fight back in the real market, even after the adversary reached the critical number, the amount of investment needed is no doubt an astronomical number. It would be a heavy burden for the enterprise even if affordable. So the beginning is always the most important part, and it's better to take the first battle as the decisive battle.

4. The finance ability is crucial. If an enterprise don't have the advantage of first action, and the finance situation is not so well, it should consider the situation seriously before stepping into the market. While any enterprise which occupies the network externality market will find the investment worthy due to the strong lock-in and monopoly.

\section{Prepare for the decisive battle in the fractionized instant massager software market in China}

In the following part, we will analyze and predict the coming fractionized instant massager (IM in short) software market in China.

\subsection{Comprehensive instant massager software market}

Typical products in this market are Tencent QQ, MSN, Sina UC and so on. The Tencent QQ have already taken the majority of the market $(>70 \%)$. Thanks to the high network externality of the IM, the large number of users of QQ gives the network enormous value and this high value resulted in a strong lock-in of users. In the conventional way of operating an IM product, any other enterprises which attempt to offset the switch cost and the opportunity cost for users to give up QQ so as to attract them to their side will meet a high investment and the fierce counterattack of Tencent Company. It can be predicted that in the near future the Tencent QQ will hold its favorable profitable position.

\subsection{Website instant massager software market}

Website IM deals mainly with the online correspondence between website members, websites and members, buyers and sellers and so on. In this new field, the TQ [6] have gained the advantage of first action. "An investigation on the representative websites of 10 open industry and the evaluation of the whole IM show that $83 \%$ of the website which adopt IM as their sales platform chose TQ as their instant massager."[7] By now the website IM software market is not fully developed, it is still in the period of accumulating early buyers as shown in the early area in the figures above. The new enterprises should come in the market quickly and competing with TQ in attracting consumers if they are to gain a favorable situation 
with a comparatively low cost. While for TQ it will be a major concern how to reach the critical number early.

\subsection{Enterprise instant massager software market}

Enterprise IM deals mainly with the online correspondence of management, communication, dealing and so on inside the enterprises and between the enterprises. There are already some kinds such as the Sametime of IBM, the ActiveMassager of Hengchuang Company. There is none which gained the advantage of first action. However the potential enormous profit of this market has attracted much attention. Once the technique and commercial condition is ready, there is sure to be a severe campaign.

There is another trend that should not be ignored: just because no enterprises have gained advantage of first action and the information system of enterprise is a high cost investment, there is a greater chance for the many kinds of new enterprise IM to realize interlinking with each other (While in other IM markets this interlinking is rare.).If so, the situation of the winner taking the majority share as shown above may not occur. The compatibility of products will result in a market carved up by a number of enterprises which have similar shares.

\subsection{Instant massager software for particular group of people.}

The "xiaonei" massager serves as a perfect case in this market. Aiming at college student has won it 3 million users. [8] Another "PPme" commercial massager calls itself "the working IM made especially for the people professional in lights." so as to attract consumers of that industry. Obviously these IM have obtained much inspiration from the success of web2.0.

There are several trends:

1. The professional aiming and the comparatively few potential users would make the spreading of the product more like the single product market above. That is, one enterprise goes in and occupies the market in a short time before the competitors come.

2. The professional aiming gives this kind of IM some advantage when competing with traditional comprehensive IM in certain professional areas and thus helping it attract many buyers back (these users chose the comprehensive IM because there were no other IMs before). This will cause a decrease in value of network of the comprehensive IM and provide a chance to break the monopoly of the Tencent QQ

3. The problem of not being interlinked will be even more serious with the kinds of IMs growing. Also it will be extravagant running several IMs on one computer. So it's an important question whether these IMs will re-combine to form a totally new standard of the industry and a new vast correspondence network based on it. If the interlinking is realized, the market will not follow the form of development of the two-product market model as above. (Because we assumed that the products are not compatible.) 


\subsection{Welcome the time of open source instant massager software.}

The P2P software sever provider-DianShi software company in Shenzhen have boldly released its ANYQ IM to enterprises for free in an open source way. This product is reputed as a million of investment, a large number of users and distributed organized. There is no doubt that this action will influence the market deeply: because the ability of interlinking with other IM, the users enjoy the value of the whole network so that the company will not have to worry about the attack led by competitors as shown in the two-product market model and even realize the development form similar to the single-product market model!

Moreover the open source ANYQ have the potential to have a larger network than any of the other products which is not open source and this larger network may be a new revolution in the IM software market.

\section{Sum-up}

Based on experimental economics method and multi-agent modeling, this essay analyzed the spreading of network externality product market, demonstrated and explored the behavior of macroscopic consumers and the important aspects which influence the procedure as well as the strategy of the enterprises; predicted the future of China's fractionized IM software market. This research also shows the advantage of experimental economics method over traditional way in dealing with dynamic procedures. This model can still be improved to simulate the market with detailed data of consumers and advertisements.

\section{Reference}

1. http://en.wikipedia.org/wiki/Metcalfe\%27s_law

2. http://adver.qq.com/dctx_04.shtml 2007-5-17

3. http://down.iresearch.cn/Graphs/Content/8842.html

4. China Internet Industry Survey Report http://report.internetdigital.org/

5. http://www.tencent.com/about/history.shtml

6. http://qtt.tq.cn/

7. http://it.sohu.com/20060913/n245324149.shtml Sohu IT

8. http://xiaonei.com/pages/im.jsp 